\title{
Phenformin Induces Caspase-dependent Apoptosis of FaDu Head and Neck Squamous Cell Carcinoma Cells
}

\author{
YO-SEOB SEO ${ }^{1}$, TAE-HYEON KIM ${ }^{2}$, HYANGI LIM ${ }^{2}$, JI-SU OH ${ }^{3}$, JAE-SEEK YOU ${ }^{3}$, \\ GYEONG-JE LEE ${ }^{4}$, SUN-KYOUNG YU ${ }^{2}$, DO KYUNG KIM ${ }^{2}$, HEUNG-JOONG KIM ${ }^{2}$, \\ CHUN SUNG KIM ${ }^{2}$, SOOK-YOUNG LEE ${ }^{5}$, SU-GWAN KIM ${ }^{3}$ and JAE-SUNG KIM ${ }^{2}$ \\ ${ }^{1}$ Department of Oral and Maxillofacial Radiology, Chosun University, Gwang-Ju, Republic of Korea; \\ ${ }^{2}$ Oral Biology Research Institute, Chosun University, Gwang-Ju, Republic of Korea; \\ ${ }^{3}$ Department of Oral and Maxillofacial Surgery, Chosun University, Gwang-Ju, Republic of Korea; \\ ${ }^{4}$ Department of Prosthodontics, School of Dentistry, Chosun University, Gwang-Ju, Republic of Korea; \\ ${ }^{5}$ Marine Bio Research Center, Chosun University, Wando-gun, Republic of Korea
}

\begin{abstract}
Background/Aim: The present study aimed to investigate the apoptotic effects of phenformin, a therapeutic agent for diabetes, on head and neck squamous cell carcinoma (HNSCC). Materials and Methods: Cytotoxicity was measured by the MTT and liveldead cell assay. Phenformin-induced apoptotic FaDu cell death and its associated cellular signaling pathways were investigated by hematoxylin and eosin staining, 4',6-diamidino-2phenylindole staining, caspase-3 activity assay, fluorescenceactivated cell sorting analysis, and western blotting. Results: Phenformin promoted death of and apoptotic processes in FaDu cells, including morphological alterations and nuclear condensation. Furthermore, treatment with phenformin increased caspase-3 activity and apoptotic populations via the caspase cascade through cleavage of capspase-8, -9, and -3 and poly(ADP-ribose) polymerase in $\mathrm{FaDu}$ cells. Moreover, phosphorylation levels of mitogen-activated protein kinases, nuclear factor- $\mathrm{KB}$, and $A K T$ were downregulated in $\mathrm{FaDu}$ cells by phenformin. Conclusion: Phenformin induced death of FaDu cells via caspasedependent extrinsic and intrinsic apoptosis pathways and is a promising novel therapeutic agent for HNSCC.
\end{abstract}

Head and neck squamous cell carcinoma (HNSCC) is the sixth most prevalent cancer $(1,2)$. Although HNSCC is unexpectedly complex in terms of pathophysiological

Correspondence to: Jae-Sung Kim, Ph.D., Pre-Dentistry, School of Dentistry, Chosun University, 309 Philmun-daero, Dong-gu, Gwangju, 61452, Republic of Korea. Tel: +82 622307362, Fax: +82 622307362, e-mail: js_kim@chosun.ac.kr

Key Words: Phenformin, head and neck squamous carcinoma, apoptosis, caspase. etiology, morphological characteristics, and clinical features, it generally arises from tissues of the oral cavity, including the oropharynx, larynx, and hypopharynx $(2,3)$. Not only are tobacco smoking and alcohol consumption traditionally implicated as representative risk factors for HNSCC (4), but infection with human papillomavirus has also recently been proven as one of its pathophysiological etiologies (5). Lymphatic metastatic spread of HNSCC significantly reduces the 5 -year overall survival rate by approximately 40 to $50 \%(6,7)$. Various clinical interventions, including surgery, radiotherapy, and chemotherapy, are performed for patients with HNSCC (8). Although there are still grave concerns in regard to the balance between drug response and adverse drug reactions, new clinical treatment strategies for HNSCC are warranted (8). However, despite advances in clinical interventions for HNSCC, the frequency of distant metastasis and the overall survival rate of patients with HNSCC, has not significantly improved over the past few decades (9). Hence, there is an urgent need to develop effective chemotherapeutic agents with minimal side-effects for patients with HNSCC.

Phenformin (CAS number 114-86-3, $\mathrm{C}_{10} \mathrm{H}_{15} \mathrm{~N}_{5}$ ), shown in Figure 1, is a biguanide with anti-diabetic activity (10). Previous preclinical and clinical studies suggested that phenformin not only has a high safety profile (11), but also shares similar mechanisms of action with metformin in inhibiting cell proliferation, and inducing cell-cycle arrest, and apoptosis of various cancer cells (12). Recently, phenformin was found to exert antitumor effects, including the suppression of cell proliferation and the induction of apoptosis in various cancer types, including breast (11), lung (13), melanoma (14), glioblastoma (15) and colon (16). Thus, the present study aimed to evaluate potential antitumorigenic effects and cellular signaling pathways that mediate the effects of phenformin in HNSCC cells. 


\section{Materials and Methods}

Cell culture. FaDu cells, a human HNSCC cell line, were obtained from the American Type Culture Collection (ATCC, Rockville, MD, USA). FaDu cells were grown in minimum essential medium (Life Technologies, Grand Island, NY, USA) containing 10\% fetal bovine serum (FBS) and were cultured in a humidified incubator at $37^{\circ} \mathrm{C}$ with $5 \% \mathrm{CO}_{2}$ according to the ATCC guidelines.

Cell viability assay. In order to determine the half-maximal inhibitory concentration $\left(\mathrm{IC}_{50}\right)$ of phenformin, FaDu cells $\left(1 \times 10^{5}\right.$ cells $/ \mathrm{ml}$ ) were cultured in 96 -well plates and treated with $0,0.25$, $0.5,1,2$, and $4 \mathrm{mM}$ phenformin for $24 \mathrm{~h}$ at $37^{\circ} \mathrm{C}$. Thereafter, the cells were incubated for a further $4 \mathrm{~h}$ with $20 \mu \mathrm{l}$ of $5 \mathrm{mg} / \mathrm{ml} \mathrm{3-(4,5-}$ dimethylthiazol-2-yl)-2,5-diphenyltetrazolium bromide (MTT) (Life Technologies). The supernatant was removed, and $200 \mu \mathrm{l} /$ well dimethyl sulfoxide were added to the samples with to dissolve the MTT crystals. The optical density (OD) of each well was measured at $570 \mathrm{~nm}$ using a spectrophotometer (Epoch microplate spectrophotometer; BioTek instruments, Winooski, VT, USA).

Cell survival assay. To verify the survival of FaDu cells treated with phenformin, cell survival assay was performed using a live/dead cell viability assay kit (ThermoFisher Scientific, Waltham, MA, USA), according to the manufacturer's instructions. Briefly, FaDu cells $\left(1 \times 10^{5}\right.$ cells $\left./ \mathrm{ml}\right)$ were cultured in an eight-well chamber slide (Sigma-Aldrich, St. Louis, MO, USA) and treated with $0,0.5$, and $1 \mathrm{mM}$ phenformin for $24 \mathrm{~h}$ at $37^{\circ} \mathrm{C}$. Thereafter, $\mathrm{FaDu}$ cells were stained with green calcein-AM to stain the live cells (green fluorescence) and with ethidium homodimer-1 to stain the dead cells (red fluorescence). The cells were imaged using a fluorescence microscope (Eclipse TE2000; Nikon Instruments, Melville, NY, USA).

Hematoxylin and eosin $(H \& E)$ staining. $\mathrm{H} \& \mathrm{E}$ staining was performed to allow observation of the morphological alterations in phenformin-treated FaDu cells. Briefly, FaDu cells $\left(1 \times 10^{5}\right.$ cells $\left./ \mathrm{ml}\right)$ were cultured in an eight-well chamber slide (Sigma-Aldrich) and then treated with $0,0.5$, and $1 \mathrm{mM}$ phenformin for $24 \mathrm{~h}$ at $37^{\circ} \mathrm{C}$. Thereafter, FaDu cells were fixed with $4 \%$ paraformaldehyde for 30 min at $4^{\circ} \mathrm{C}$ and $\mathrm{H} \& \mathrm{E}$ staining was performed. The cells were observed and imaged using a microscope (Leica DM750, Leica Microsystems, Heerbrugg, Switzerland) equipped with a $3 \mathrm{MP}$ Color Diagnostic Monitor (CX30p, Wide, Yongin, Republic of Korea).

Nuclear staining. To determine whether phenformin induced nuclear condensation in FaDu cells, 4',6-diamidino-2-phenylindole (DAPI) staining was performed. Briefly, FaDu cells $\left(1 \times 10^{5}\right.$ cells $\left./ \mathrm{ml}\right)$ were cultured in an eight-well chamber slide (Sigma-Aldrich) and treated with $0,0.5$, and $1 \mathrm{mM}$ phenformin for $24 \mathrm{~h}$ at $37^{\circ} \mathrm{C}$. Thereafter, cells were stained with $1 \mathrm{mg} / \mathrm{ml}$ DAPI (Sigma-Aldrich) for $20 \mathrm{~min}$. Nuclear condensation was observed and imaged using a fluorescence microscope (Eclipse TE200; Nikon Instruments).

Caspase-3/-7 activity assay. To determine whether phenformin increases the activity of caspase-3/-7 in FaDu cells, caspase-3/-7 activity was assayed using the cell-permeable fluorogenic substrate PhiPhiLux- $\mathrm{G}_{1} \mathrm{D}_{2}$ kit (OncoImmunin Inc.; Gaithersburg, MD, USA) according to the manufacturer's instructions. Cells were imaged using fluorescence microscopy (Eclipse TE200; Nikon Instruments).
Fluorescence-activated cell sorting analysis (FACS). In order to verify whether phenformin increases the proportion of apoptotic FaDu cells, FACS was performed using annexin V-fluorescein isothiocyanate (FITC) and propidium iodide (PI) (Cell Signaling Technology, Danvers, MA, USA). Briefly, FaDu cells $\left(5 \times 10^{5}\right.$ cells $\left./ \mathrm{ml}\right)$ were cultured on a sixwell plate for $24 \mathrm{~h}$, after which cells were treated with $0,0.5$, and 1 $\mathrm{mM}$ phenformin for $24 \mathrm{~h}$. Thereafter, the collected $\mathrm{FaDu}$ cells were stained with annexin V-FITC and PI and incubated for $15 \mathrm{~min}$ at $37^{\circ} \mathrm{C}$. Afterwards, the apoptotic populations were analyzed using BD Cell Quest ${ }^{\circledR}$ version 3.3 (Becton Dickinson, San José, CA, USA).

Western blot analysis. FaDu cells $\left(5 \times 10^{5}\right.$ cells $\left./ \mathrm{ml}\right)$ cultured on a six-well plate were treated with $0,0.5$, and $1 \mathrm{mM}$ phenformin for $24 \mathrm{~h}$. Thereafter, cell lysates were prepared using a cell lysis buffer (Cell Signaling Technology) according to the manufacturer's instructions. Protein concentrations were measured by performing the bicinchoninic acid protein assay (Thermo Scientific, Rockford, IL, USA). Equal amounts of the cell lysates were then electrophoresed by sodium dodecyl sulfate polyacrylamide gel electrophoresis and then analyzed by western blotting using antibodies to Fas ligand (FASL, $40 \mathrm{kDa}$; Cell Signaling Technology), caspase-8 (18, 43, and $57 \mathrm{kDa}$; Cell Signaling Technology), BH3-interacting domain death agonist (BID, $20 \mathrm{kDa}$; Santa Cruz Biotechnology Inc., Dallas, TX, USA), B-cell lymphoma-2 (BCL2, 26 $\mathrm{kDa}$, Santa Cruz Biotechnology Inc.), BCL-extra large (BCL-xL, 30 $\mathrm{kDa}$; Cell Signaling Technology), BCL2-associated death promoter (BAD, $23 \mathrm{kDa}$; Cell Signaling Technology), BCL2-associated X protein (BAX, 20 kDa; Cell Signaling Technology), p53 (53 kDa; Cell Signaling Technology), cleaved caspase-3 (17 and $19 \mathrm{kDa}$; Cell Signaling Technology), poly(ADP-ribose) polymerase (PARP, 89 and $116 \mathrm{kDa}$; Cell Signaling Technology), $\beta$-actin (45 kDa; Santa Cruz Biotechnology Inc.), phospho-extracellular signal-regulated kinase 1/2 (ERK1/2, 42 and $44 \mathrm{kDa}$; Santa Cruz Biotechnology Inc.), total ERK (42 and $44 \mathrm{kDa}$; Santa Cruz Biotechnology Inc.), phospho-p38 (38 kDa; Santa Cruz Biotechnology Inc.), total p-38 (38 kDa; Santa Cruz Biotechnology Inc.), phospho-protein kinase B (AKT, $60 \mathrm{kDa}$; Cell Signaling Technology), total-AKT (60 kDa; Cell Signaling Technology), phospho-nuclear factor kappa B (NFkB, 65 kDa; Cell Signaling Technology), and total NFkB (65 kDa; Cell Signaling Technology). The immunoreactive bands were visualized using the ECL System (Amersham Biosciences, Piscataway, NJ, USA), exposed on radiographic film or MicroChemi 4.2 (Dong-Il SHIMADZE Crop., Seoul, Korea).

Caspase-dependent cell survival assay. $\mathrm{FaDu}$ cells $\left(1 \times 10^{5}\right.$ cells $\left./ \mathrm{ml}\right)$ cultured in a 96-well plate for $24 \mathrm{~h}$. After incubation, FaDu cells were treated with $1 \mathrm{mM}$ phenformin in the presence or absence of the cellpermeant pan-caspase inhibitor carbobenzoxy-valyl-alanyl-aspartyl-(Omethyl)-fluoromethylketone (Z-VAD-fmk; Santa Cruz Biotechnology Inc.) and were incubated for $24 \mathrm{~h}$. Thereafter, western blotting of caspase-3 and MTT assay were conducted to verify whether phenformin-induced $\mathrm{FaDu}$ cell death is mediated by the caspase cascade.

Statistical analysis. Experimental data are presented as the mean \pm standard deviation of at least three independent experiments and were analyzed by analysis of variance, followed by Student's $t$-tests. Values of $p<0.05$ were considered statistically significant.

\section{Results}

The MTT assay was performed to verify whether phenformin promotes cell cytotoxicity. As shown in Figure 2A, FaDu cells 


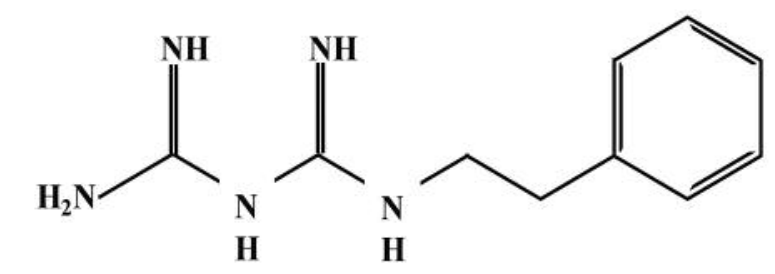

\section{Phenformin}

- CAS number: $114-86-3$

- IUPAC name: 2-(N-Phenethylcarbamimidoyl)guanidine

- Formula: $\mathrm{C}_{10} \mathrm{H}_{15} \mathrm{~N}_{5}$

- Molecular weight: 205.26

Figure 1. Chemical structure of phenformin and other relevant information.

treated with $0.25,0.5,1,2$, and $4 \mathrm{mM}$ phenformin for $24 \mathrm{~h}$ increased cell cytotoxicity in a dose-dependent manner by $8.8 \pm 2.5 \%(p<0.05), 13.7 \pm 4.7 \%(p<0.05), 27.8 \pm 1.3 \%(p<0.01)$, $67.2 \pm 0.9 \%(p<0.01)$, and $79.3 \pm 1.6 \%(p<0.01)$ relative to the untreated control. Furthermore, the $\mathrm{IC}_{50}$ value of phenformin in $\mathrm{FaDu}$ cells value was estimated to be approximately $1.5 \mathrm{mM}$ phenformin. However, to confirm that phenformin induced FaDu cell death by increasing cytotoxicity, live and dead cells were stained using a live/dead cell assay kit, in which cellpermeable green calcein AM stains live cells to produce green fluorescence, while dead cells are stained with ethidium homodimer- 1 and produce red fluorescence. As shown in Figure 3A, the number of FaDu cells was reduced by treatment with 0.5 and $1 \mathrm{mM}$ phenformin compared to those of the control. Furthermore, phenformin increased the number of dead $\mathrm{FaDu}$ cells in a dose-dependent manner compared to the controls. Taken together, our results indicate that phenformin induces cell death in a dose-dependent manner in FaDu cells.

To determine whether phenformin-mediated FaDu cell death is involved in apoptosis, the morphological alterations of $\mathrm{FaDu}$ cells treated with phenformin were observed with $\mathrm{H} \& \mathrm{E}$ staining. As shown in Figure 3B, phenformin not only reduced the number of $\mathrm{FaDu}$ cells, which is consistent with the results of live/dead cell assay, but also increased the number of $\mathrm{FaDu}$ cells with altered morphology, including cell shrinkage, which is closely associated with apoptotic cell death. Next, results of DAPI staining showed that phenformin promoted nuclear condensation in $\mathrm{FaDu}$ cells, as shown in Figure 3C. Furthermore, phenformin treatment increased the activity of capspase-3/-7 in FaDu cells in a dose-dependent manner, as shown as Figure 3D. Next, in order to verify whether phenformin induced apoptosis of FaDu cells, FACS analysis was performed using annexin-V-FITC and PI. Results of FACS analysis, as shown as Figure 3E, showed that the total proportion of dead cells was approximately $15.95 \%$ and $39.2 \%$ in $\mathrm{FaDu}$

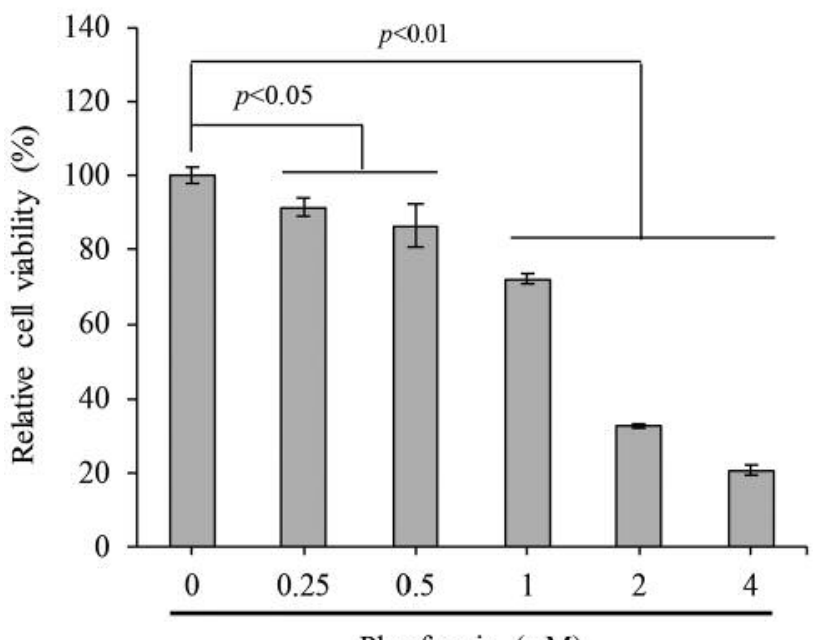

Figure 2. Phenformin increased cytotoxicity accompanied by morphological alterations in FaDu cells. Phenformin reduced cell viability of FaDu cells in a dose-dependent manner. FaDu cells were plated and subsequently treated with $0.25,0.5,1,2$, and $4 \mathrm{mM}$ phenformin for $24 \mathrm{~h}$. Thereafter, 3(4,5-dimethylthiazol-2-yl)-2,5-diphenyltetrazolium bromide assay was performed to measure the viability of FaDu cells.

cells treated with 0.5 and $1 \mathrm{mM}$ phenformin, respectively. Among these, cells in the early and late stages of apoptosis were verified to comprise $12.1 \%$ and $21.52 \%$, respectively, in $\mathrm{FaDu}$ cells treated with 0.5 and $1 \mathrm{mM}$ phenformin. Taken together, the above findings consistently indicated that apoptosis is involved in phenformin-induced $\mathrm{FaDu}$ cell death.

Next, to investigate phenformin-induced apoptosis pathways in FaDu cells, we performed western blotting using specific antibodies to proteins associated with the extrinsic and intrinsic apoptosis pathways. As shown in Figure 4A, phenformin up-regulated FASL (40 kDa) expression in $\mathrm{FaDu}$ cells in a dose-dependent manner. Phenformin treatment led to a dose-dependent increase in the protein level of cleaved caspase-8 (18 and $43 \mathrm{kDa}$ ), a downstream substrate of FASL, in $\mathrm{FaDu}$ cells. Furthermore, the up-regulation of cleaved caspase- 8 the activation of caspase-3 (17 and $19 \mathrm{kDa})$ and PARP ( $89 \mathrm{kDa}$ ) in FaDu cells treated with phenformin, as shown in Figure 4C. Hence, the above findings indicate that phenformin-induced cell death is mediated by the extrinsic apoptosis pathway, which involves the cascade activation of caspase-8, caspase-3, and PARP via FASL up-regulation in FaDu cells. Furthermore, phenformin down-regulated the expression of BID (20 kDa) in a dose-dependent manner. The expression levels of anti-apoptotic factors BCL2 $(26 \mathrm{kDa})$ and BCL-xL (30 kDa), were down-regulated by phenformin in a dose-dependent manner (Figure 4B). On the other hand, phenformin treatment induced a dose-dependent increase in the expression levels of pro-apoptotic factors BAD (23 kDa) and BAX (20 kDa), and the tumor-suppressor p53 (53 kDa) 


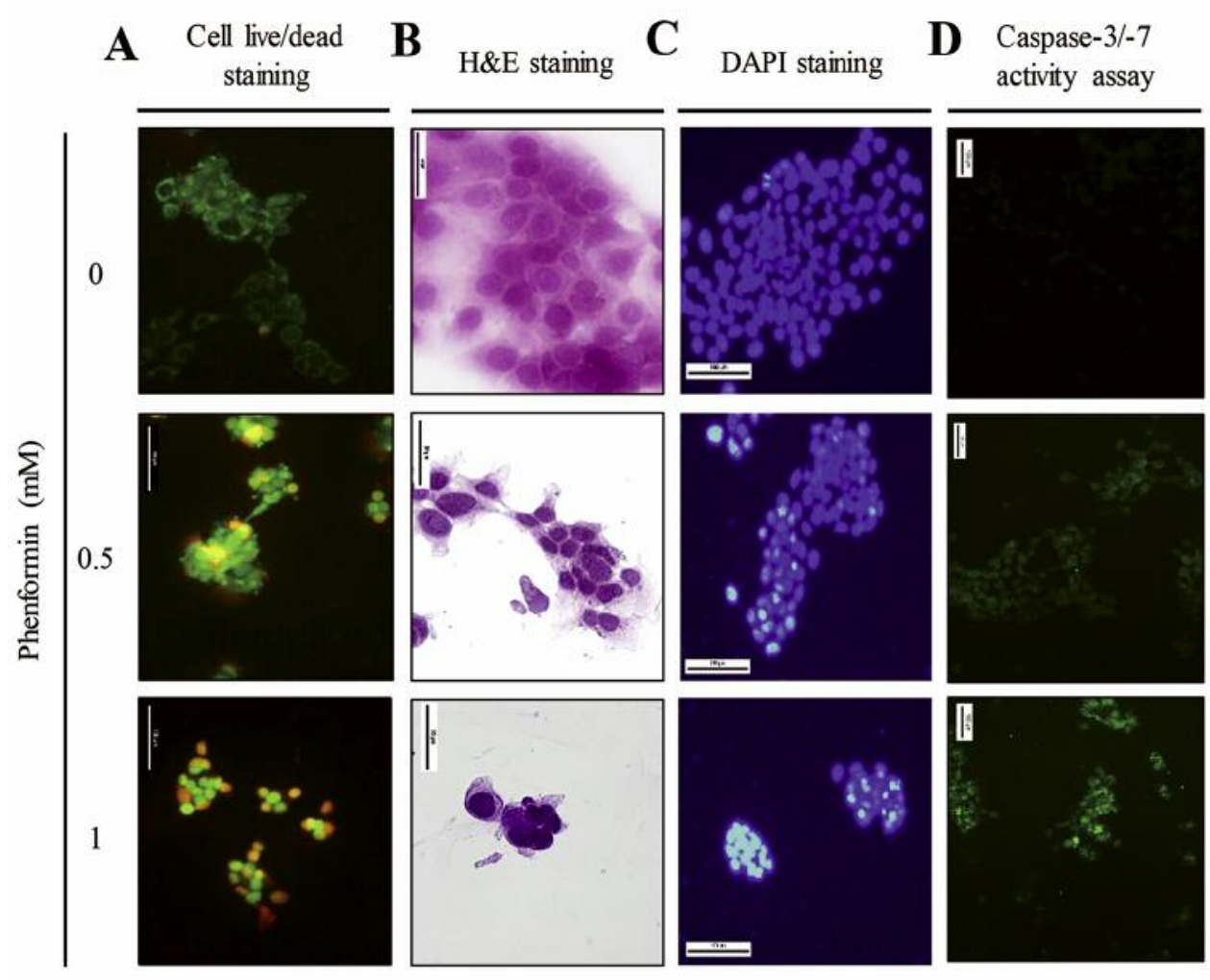

$\mathbf{E}$

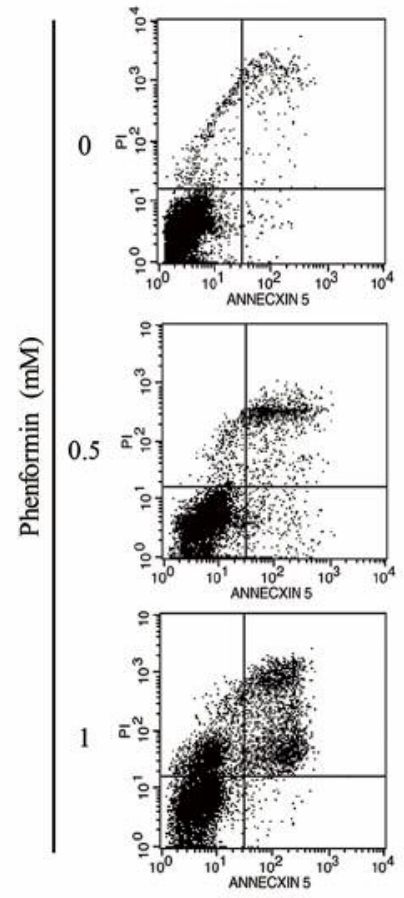

$\square$ Necrosis

口Late stage of apoptosis

-Early stage of apoptosis

口Live cells

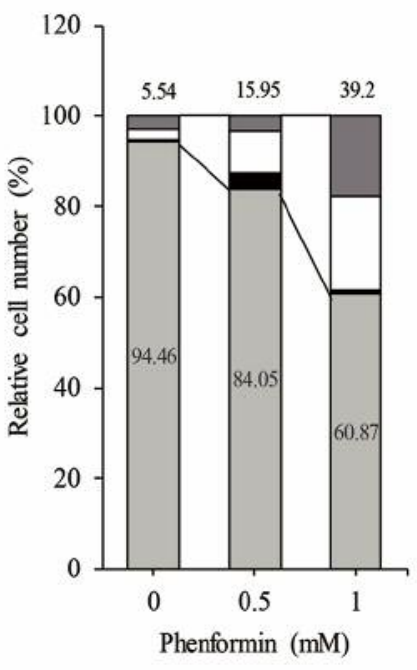

Figure 3. Phenformin induced apoptotic cell death of FaDu cells. FaDu cells were plated and then treated with 0.5 and $1 \mathrm{mM}$ phenformin for $24 \mathrm{~h}$. Thereafter, Cell live/dead assay (A), hematoxylin and eosin ( $H \&$ E) staining (B), 4',6-diamidino-2-phenylindole (DAPI) staining (C) and caspase3/-7 activity assay (D) were performed as described in Materials and Methods. A: Phenformin not only reduced the cell number, but also increased the number of dead FaDu cells (red florescence). B: FaDu cells with altered morphology, including shrinkage, were observed following phenformin treatment. C: The number of FaDu cells with condensed nuclei was increased by phenformin treatment. D: Activity of caspase-3 in FaDu cells was increased by phenformin treatment in a dose-dependent manner. E: Phenformin treatment increased apoptotic populations of FaDu cells in a dosedependent manner. FaDu cells were plated and subsequently treated with 0.5 and $1 \mathrm{mM}$ phenformin for $24 \mathrm{~h}$. Thereafter, fluorescence-activated cell sorting analysis (FACS) analysis was performed using annexin V-fluorescein isothiocyanate (FITC) and propidium iodide (PI). 
A
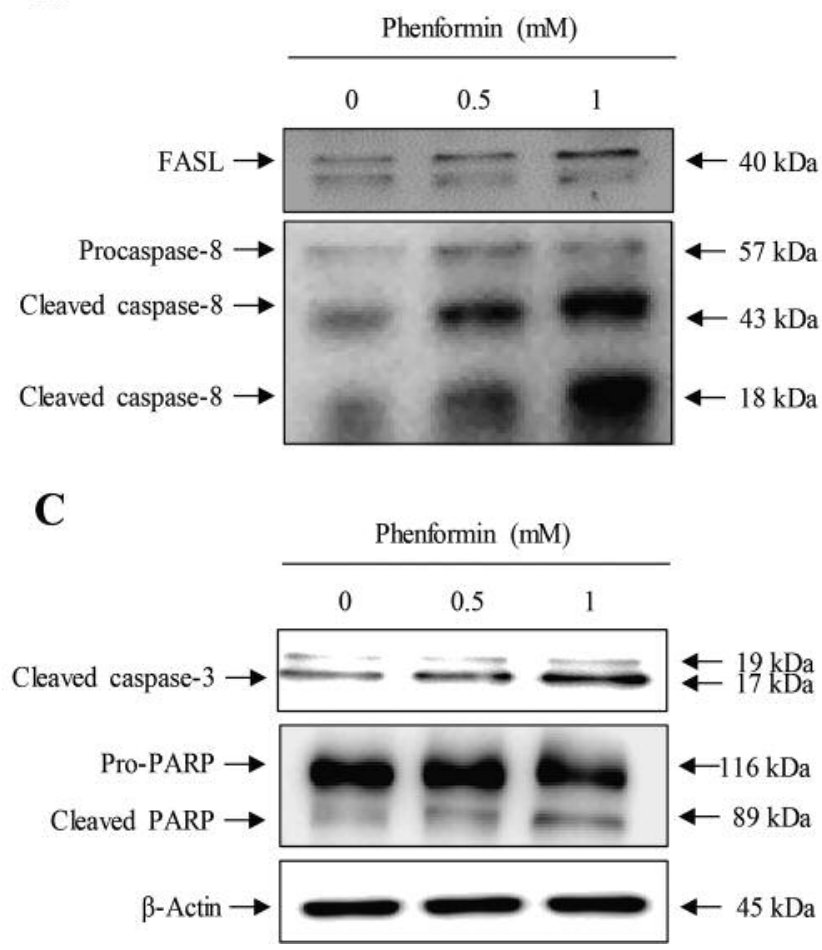

B

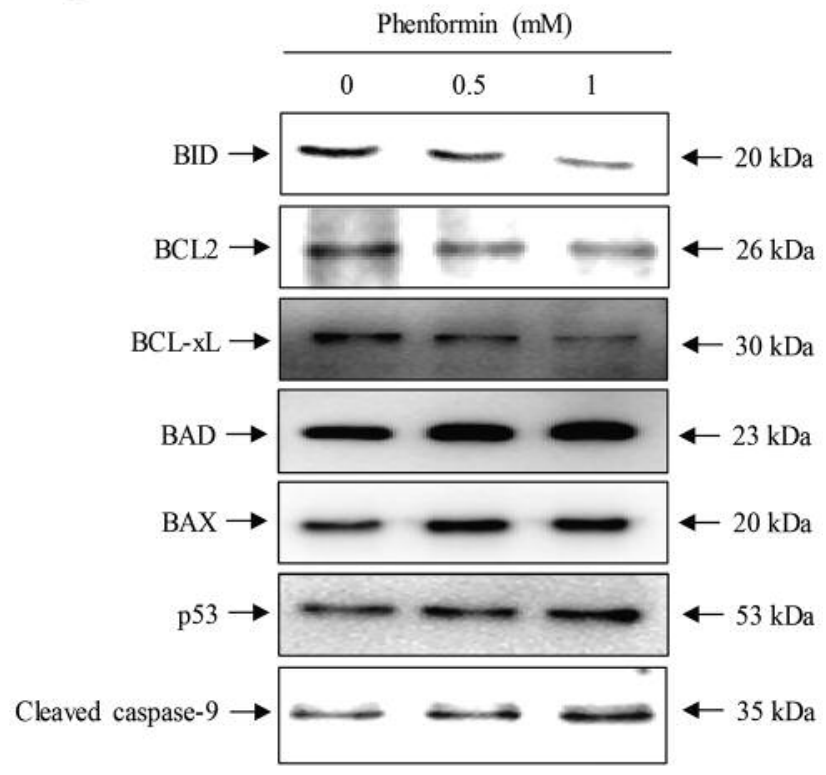

Figure 4. Phenformin-induced apoptosis of FaDu cells is mediated by FAS ligand (FASL)-triggered extrinsic and intrinsic apoptosis pathways. Western blotting was performed using specific antibodies associated with extrinsic and intrinsic apoptosis pathways in FaDu cells treated with 0.5 and $1 \mathrm{mM}$ phenformin for $24 \mathrm{~h}$. A: Phenformin-induced FaDu cell death was mediated by the extrinsic apoptosis pathway through the up-regulation of FASL and caspase-8, a pro-apoptotic factor. B: Up-regulation of cleaved caspase-8 induced the mitochondria-dependent intrinsic apoptosis pathways via reduction of BH3-interacting domain death agonist (BID) in FaDu cells treated with phenformin. C: Phenformin increased the activation of poly(ADP-ribose) polymerase (PARP) through the activation of caspase-3, a downstream target of cleaved caspase-8 and cleaved caspase-9, in FaDu cells. BCL2: B-Cell lymphoma-2; BAX: BCL2-associated X protein; BAD: BCL2-associated death promoter.

in FaDu cells. Phenformin also increased the protein level of cleaved caspase-9 (17 and $38 \mathrm{kDa}$ ) in a dose-dependent manner. Hence, our findings indicate that phenformin-induced $\mathrm{FaDu}$ cell death also involves the mitochondria-dependent intrinsic apoptosis pathway.

Next, to verify whether phenformin-induced FaDu cell death is mediated by the caspase cascade, we conducted western blotting of capsase-3 and MTT assay on FaDu cells treated with phenformin in the presence or absence of the cell-permeant pancaspase inhibitor Z-VAD-fmk. As shown in Figure 5A, although the cleaved caspase- 3 level was up-regulated in $\mathrm{FaDu}$ cells treated with $1 \mathrm{mM}$ phenformin, it cleaved caspase-3 levels were lower in the presence of $20 \mu \mathrm{M} Z$-VAD-fmk. Furthermore, the viability of $\mathrm{FaDu}$ cells significantly decreased $(p<0.01)$ relative to those of control cells as shown in Figure $5 \mathrm{~B}$. On the other hand, Z-VAD-fmk increased the viability of $\mathrm{FaDu}$ cells by approximately $23 \%(p<0.01)$ in the presence of $1 \mathrm{mM}$ phenformin. Taken together, the above findings indicate that phenformin-induced FaDu cell death is dependent on the activation of the caspase cascade.
Next, to investigate the cellular signaling pathways related to phenformin-induced apoptosis, we performed western blotting using specific antibodies to mitogen-activated protein kinases (MAPKs), NFkB, and AKT, as shown in Figure 6. Interestingly, phenformin reduced the phospho-rylation levels of ERK1/2, p38, $\mathrm{NFKB}$, and $\mathrm{AKT}$ in $\mathrm{FaDu}$ cells in a dose-dependent manner.

\section{Discussion}

In present study, the results showed that phenformin-induced FaDu cell death is mediated by caspases via the FASL-triggered extrinsic and intrinsic apoptosis pathways. Furthermore, phenformin-induced FaDu cell death is closely involved in alterations in MAPK, including the ERK1/2 and p38, NFkB, and AKT signaling pathways.

Phenformin is a biguanide with anti-diabetic activity that is almost 50-fold more potent than metformin, and is the most widely prescribed treatment for type II diabetes $(17,18)$. Metformin is a biguanide drug that has a similar chemical structure and similar pharmacological effects to those of 
A

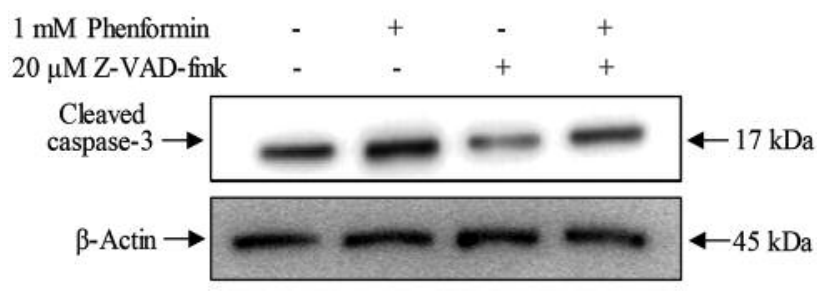

B

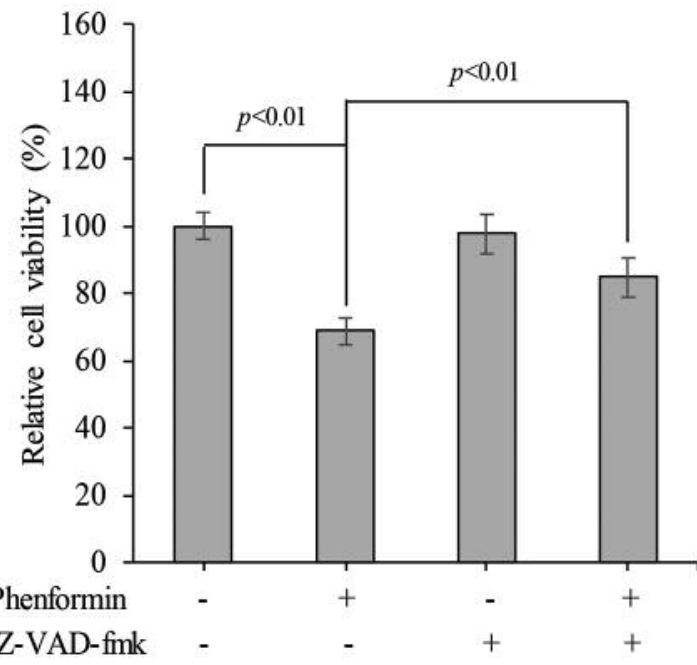

Figure 5. Phenformin-induced FaDu cell death is dependent on caspase activation. FaDu cells were plated and subsequently treated with $1 \mathrm{mM}$ phenformin in the presence or absence of $20 \mu M$ cell-permeant pan caspase inhibitor Z-VAD-fmk carbobenzoxy-valyl-alanyl-aspartyl-(Omethyl)-fluoromethylketone for $24 \mathrm{~h}$. Thereafter, western blotting (A) and MTT assay $(B)$ were performed to verify changes in caspase-3 levels and cell viability, respectively. Z-VAD-fmk inhibited the expression of cleaved caspase-3 in FaDu cells treated with phenformin. Viability of FaDu cells was increased by treatment with Z-VAD-fmk in the presence of phenformin.

phenformin $(19,20)$. Although the anticancer efficacy of metformin in diabetic patients remains to be investigated, epidemiological studies have suggested that metformin reduces incidence and risk of types of cancer (21), including gastric, colorectal (22), pancreatic (23), and lung cancer (24). Furthermore, recent studies reported that metformin suppressed cell proliferation $(25,26)$, migration $(27)$, and angiogenesis $(11$, 28) and induces cell arrest (29) and apoptosis (30) in various types of cancer cell. Currently, clinical trials that investigate metformin-induced anticancer efficacy in various types of cancer were conducted (31).

Apoptosis, also known as programmed cell death, is generally characterized by distinct morphological characteristics, including cellular shrinkage, condensation and margination of the chromatin, ruffling plasma membrane, and the formation of apoptotic bodies $(32,33)$. Apoptosis is a crucial cellular mechanism that maintains tissue homeostasis via the elimination

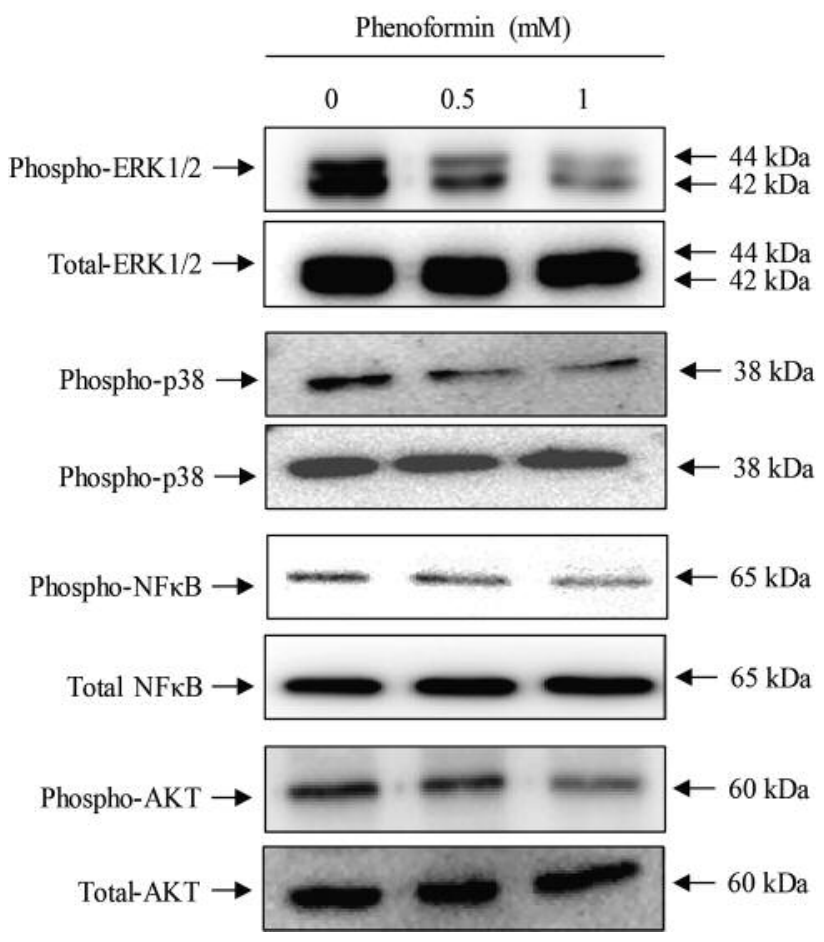

Figure 6. Phenformin reduced the phosphorylation of proteins in the mitogen-activated protein kinases (MAPKs), nuclear factor-kappa $B$ $(N F K B)$, and phospho-protein kinase $B(A K T)$ pathways in FaDu cells. $\mathrm{FaDu}$ cells were plated and then treated with 0.5 and $1 \mathrm{mM}$ phenformin for $24 \mathrm{~h}$. Thereafter, western blotting using specific antibodies against extracellular signal-regulated kinase 1/2 (ERK1/2), p38, NFkB, and $A K T$ was performed to verify potential cellular signaling pathways associated with phenformin-induced apoptosis.

of harmful or unnecessary cells (34). Hence, there is an urgent need to develop novel chemotherapeutic agents that can trigger cancer-specific cell death such as via apoptosis (34). Interestingly, recent studies reported that phenformin, which has similar chemical structure and physiological effects to those of metformin, not only inhibits cell proliferation and angiogenesis, but also induces cell arrest and apoptosis in various cancer types, including cholangiocarcinoma, breast, and non-small cell lung cancer $(11,13,35-37)$. Consistent with the findings of previous studies associated with phenformin-induced cancer cell apoptosis, phenformin-induced $\mathrm{FaDu}$ cell death was found to be mediated by apoptotic processes, such as cellular shrinkage and nuclear condensation (Figure 2, and 3A-C). Furthermore, our results based on the caspase-3/-7 activity assay using cellpermeable fluorogenic substrate PhiPhiLux- $\mathrm{G}_{1} \mathrm{D}_{2}$ kit (OncoImmunin Inc., Gaithersburg, MD, USA) and FACS analysis using PI and annexin-V showed that phenformin increased the activity of caspase-3 (Figure 3D) and the proportion of apoptotic FaDu cells (Figure 3E). In particular, caspase-3 is a key pro-apoptotic factor that induces apoptotic cell death by catalyzing the cleavage of specific cellular proteins 
(38). Therefore, these findings indicated that phenformin-induced $\mathrm{FaDu}$ cell death involves apoptosis. Generally, cellular mechanisms underlying apoptosis can be classified into the extrinsic death receptor-mediated and the intrinsic mitochondriadependent pathways (33). These are closely regulated by apoptotic initiators caspase- 8 and -9 of the extrinsic and intrinsic apoptosis pathways, respectively. Furthermore, the activation of both caspase- 8 and -9 can induce the 'executive' caspases include caspase-3, -6, and -7 (39). Hence, the activation of caspases has being considered as an important chemotherapeutic strategy. As shown in Figure 4, phenformin-induced FaDu cell death was mediated by the activation of caspase- 3 through the activation of apoptotic initiators caspase- 8 and -9 located on extrinsic death receptor-mediated and intrinsic mitochondriadependent pathways, respectively. Furthermore, Figure 5 showed that treatment with the pan-caspase inhibitor Z-VAD-fmk not only counteracted phenformin-induced $\mathrm{FaDu}$ cell death, but also suppressed the expression of cleaved caspase- 3 in phenformintreated $\mathrm{FaDu}$ cells. These findings demonstrated that phenformin induced a caspase-dependent apoptotic cell death of FaDu cells through the extrinsic death receptor-mediated and intrinsic mitochondria-dependent pathways.

Recent studies that investigated the anticancer effects of phenformin reported the suppression of ERK phosphorylation in tumor suppressor neurofibromin-mutant melanoma (40) and breast cancer cells (36). Furthermore, Wang et al. reported that phenformin inhibited cell growth and angiogenesis through the suppression of ERK and AKT phosphorylation in non-small cell lung cancer cells (13). Similarly to previous studies, phenformin suppressed the phosphorylation of MAPKs ERK, p38, NFKB, and $\mathrm{AKT}$ in $\mathrm{FaDu}$ cells. Hence, these cellular signaling pathways appear to be closely associated with phenformin-induced apoptosis of $\mathrm{FaDu}$ cells.

In conclusion, our findings demonstrated that phenformin induces caspase-dependent FaDu cell death via FASL-induced extrinsic death receptor-mediated and intrinsic mitochondriadependent apoptosis. Hence, our results highlighted the potential use of phenformin and other biguanides as HNSCC therapeutics.

\section{Conflicts of Interest}

The Authors have no conflicts of interest.

\section{Authors' Contributions}

Y.S.S., T.H.K., H.L., and J.S.K. contributed to the experimental work; All authors including J.S.O., J.S.Y., G.J.L., S.K.Y., D.K.K., H.J.K., C.S.K., S.Y.L., S.G.W., and J.S.K. participated in data analysis and interpretations; Y.S.S. and J.S.K drafted the article. All Authors gave final approval for publication.

\section{Acknowledgements}

This study was supported by research fund from Chosun University Dental Hospital, 2018.

\section{References}

1 Parkin DM, Bray F, Ferlay J and Pisani P: Global cancer statistics, 2002. CA Cancer J Clin 55(2): 74-108, 2005. PMID: 15761078. DOI: 10.3322/canjclin.55.2.74

2 Porcheri C, Meisel CT and Mitsiadis T: Multifactorial contribution of notch signaling in head and neck squamous cell carcinoma. Int $\mathrm{J}$ Mol Sci 20(6), 2019. PMID: 30917608. DOI: 10.3390/ijms20061520

3 Ausoni S, Boscolo-Rizzo P, Singh B, Da Mosto MC, Spinato G, Tirelli G, Spinato R and Azzarello G: Targeting cellular and molecular drivers of head and neck squamous cell carcinoma: Current options and emerging perspectives. Cancer Metastasis Rev 35(3): 413-426, 2016. PMID: 27194534. DOI: 10.1007/ s10555-016-9625-1

4 Castellsague X, Quintana MJ, Martinez MC, Nieto A, Sanchez MJ, Juan A, Monner A, Carrera M, Agudo A, Quer M, Munoz N, Herrero R, Franceschi S and Bosch FX: The role of type of tobacco and type of alcoholic beverage in oral carcinogenesis. Int J Cancer 108(5): 741-749, 2004. PMID: 14696101. DOI: 10.1002/ijc.11627

5 Snow AN and Laudadio J: Human papillomavirus detection in head and neck squamous cell carcinomas. Adv Anat Pathol 17(6): 394403, 2010. PMID: 20966645. DOI: 10.1097/ PAP.0b013e3181f895c1

6 Brockstein B, Haraf DJ, Rademaker AW, Kies MS, Stenson KM, Rosen F, Mittal BB, Pelzer H, Fung BB, Witt ME, Wenig B, Portugal L, Weichselbaum RW and Vokes EE: Patterns of failure, prognostic factors and survival in locoregionally advanced head and neck cancer treated with concomitant chemoradiotherapy: A 9-year, 337-patient, multi-institutional experience. Ann Oncol 15(8): 1179-1186, 2004. PMID: 152772 56. DOI: 10.1093/annonc/mdh308

7 Leemans CR, Braakhuis BJ and Brakenhoff RH: The molecular biology of head and neck cancer. Nat Rev Cancer 11(1): 9-22, 2011. PMID: 21160525. DOI: $10.1038 / \mathrm{nrc} 2982$

8 Ruwali M, Dhawan A, Pant MC, Rahman Q, Khurana SM and Parmar D: Clinical management of head and neck cancer cases: Role of pharmacogenetics of cyp2 and gsts. Oncol Res Treat 39(4): 221226, 2016. PMID: 27160276. DOI: 10.1159/00044 4608

9 Mandapathil M, Roessler M, Werner JA, Silver CE, Rinaldo A and Ferlito A: Salvage surgery for head and neck squamous cell carcinoma. Eur Arch Otorhinolaryngol 271(7): 1845-1850, 2014. PMID: 24756615. DOI: 10.1007/s00405-014-3043-1

10 Ohnishi S, Mizutani H and Kawanishi S: The enhancement of oxidative DNA damage by anti-diabetic metformin, buformin, and phenformin, via nitrogen-centered radicals. Free Radic Res 50(8): 929-937, 2016. PMID: 27328723. DOI: 10.1080/1071 5762.2016 .1204651

11 Orecchioni S, Reggiani F, Talarico G, Mancuso P, Calleri A, Gregato G, Labanca V, Noonan DM, Dallaglio K, Albini A and Bertolini F: The biguanides metformin and phenformin inhibit angiogenesis, local and metastatic growth of breast cancer by targeting both neoplastic and microenvironment cells. Int J Cancer 136(6): E534-544, 2015. PMID: 25196138. DOI: 10.1002/ijc.29193

12 Zhang HH and Guo XL: Combinational strategies of metformin and chemotherapy in cancers. Cancer Chemother Pharmacol 78(1): 1326, 2016. PMID: 27118574. DOI: 10.1007/s00280-016-3037-3

13 Wang ZD, Wei SQ and Wang QY: Targeting oncogenic KRAS in non-small cell lung cancer cells by phenformin inhibits growth and angiogenesis. Am J Cancer Res 5(11): 3339-3349, 2015. PMID: 26807315.

14 Petrachi T, Romagnani A, Albini A, Longo C, Argenziano G, Grisendi G, Dominici M, Ciarrocchi A and Dallaglio K: Therapeutic potential of the metabolic modulator phenformin in targeting the stem 
cell compartment in melanoma. Oncotarget 8(4): 6914-6928, 2017. PMID: 28036292. DOI: 10.18632/ oncotarget.14321

15 Jiang W, Finniss S, Cazacu S, Xiang C, Brodie Z, Mikkelsen T, Poisson L, Shackelford DB and Brodie C: Repurposing phenformin for the targeting of glioma stem cells and the treatment of glioblastoma. Oncotarget 7(35): 56456-56470, 2016. PMID: 27486821. DOI: $10.18632 /$ oncotarget.10919

16 Lea MA, Chacko J, Bolikal S, Hong JY, Chung R, Ortega A and desbordes C: Addition of 2-deoxyglucose enhances growth inhibition but reverses acidification in colon cancer cells treated with phenformin. Anticancer Res 31(2): 421-426, 2011. PMID: 21378320.

17 McGuinness ME and Talbert RL: Phenformin-induced lactic acidosis: A forgotten adverse drug reaction. Ann Pharmacother 27(10): 11831187, 1993. PMID: 8251683. DOI: 10.1177/ 106002809302701004

18 Jackson AL, Sun W, Kilgore J, Guo H, Fang Z, Yin Y, Jones HM, Gilliam TP, Zhou C and Bae-Jump VL: Phenformin has antitumorigenic effects in human ovarian cancer cells and in an orthotopic mouse model of serous ovarian cancer. Oncotarget $8(59)$ : 100113-100127, 2017. PMID: 29245964. DOI: 10.18632/ oncotarget.22012

19 Janzer A, German NJ, Gonzalez-Herrera KN, Asara JM, Haigis MC and Struhl K: Metformin and phenformin deplete tricarboxylic acid cycle and glycolytic intermediates during cell transformation and NTPs in cancer stem cells. Proc Natl Acad Sci USA 111(29): 1057410579, 2014. PMID: 25002509. DOI: 10.1073/pnas.1409844111

20 Kowall B, Stang A, Rathmann W and Kostev K: No reduced risk of overall, colorectal, lung, breast, and prostate cancer with metformin therapy in diabetic patients: Database analyses from germany and the UK. Pharmacoepidemiol Drug Saf 24(8): 865-874, 2015. PMID: 26132313. DOI: $10.1002 /$ pds.3823

21 Evans JM, Donnelly LA, Emslie-Smith AM, Alessi DR and Morris AD: Metformin and reduced risk of cancer in diabetic patients. BMJ 330(7503): 1304-1305, 2005. PMID: 15849206. DOI: 10.1136/bmj.38415.708634.F7

22 Zhang ZJ, Zheng ZJ, Kan H, Song Y, Cui W, Zhao G and Kip KE: Reduced risk of colorectal cancer with metformin therapy in patients with type 2 diabetes: A meta-analysis. Diabetes Care 34(10): 23232328, 2011. PMID: 21949223. DOI: 10.2337/ dc11-0512

23 Wang Z, Lai ST, Xie L, Zhao JD, Ma NY, Zhu J, Ren ZG and Jiang GL: Metformin is associated with reduced risk of pancreatic cancer in patients with type 2 diabetes mellitus: A systematic review and meta-analysis. Diabetes Res Clin Pract 106(1): 19-26, 2014. PMID: 24837144. DOI: 10.1016/j.diabres. 2014.04.007

24 Zhang ZJ, Bi Y, Li S, Zhang Q, Zhao G, Guo Y and Song Q: Reduced risk of lung cancer with metformin therapy in diabetic patients: A systematic review and meta-analysis. Am J Epidemiol 180(1): 11-14, 2014. PMID: 24920786. DOI: 10. 1093/aje/kwu124

25 Kato K, Gong J, Iwama H, Kitanaka A, Tani J, Miyoshi H, Nomura K, Mimura S, Kobayashi M, Aritomo Y, Kobara H, Mori H, Himoto T, Okano K, Suzuki Y, Murao K and Masaki T: The antidiabetic drug metformin inhibits gastric cancer cell proliferation in vitro and in vivo. Mol Cancer Ther 11(3): 549-560, 2012. PMID: 22222629. DOI: 10.1158/1535-7163.MCT-11-0594

26 Wang Y, Dai W, Chu X, Yang B, Zhao M and Sun Y: Metformin inhibits lung cancer cells proliferation through repressing microrna222. Biotechnol Lett 35(12): 2013-2019, 2013. PMID: 23974492. DOI: $10.1007 / \mathrm{s} 10529-013-1309-0$

27 Qiang P, Shao Y, Sun YP, Zhang J and Chen LJ: Metformin inhibits proliferation and migration of endometrial cancer cells through regulating PI3K/AKT/MDM2 pathway. Eur Rev Med
Pharmacol Sci 23(4): 1778-1785, 2019. PMID: 30840303. DOI: 10.26355/eurrev_201902_17140

28 Gao S, Jiang J, Li P, Song H, Wang W, Li C and Kong D: Attenuating tumour angiogenesis: A preventive role of metformin against breast cancer. Biomed Res Int 2015: 592523, 2015. PMID: 25883966. DOI: $10.1155 / 2015 / 592523$

29 Zhao L, Wen ZH, Jia CH, Li M, Luo SQ and Bai XC: Metformin induces $\mathrm{g} 1$ cell cycle arrest and inhibits cell proliferation in nasopharyngeal carcinoma cells. Anat Rec 294(8): 1337-1343, 2011. PMID: 21717584. DOI: $10.1002 /$ ar.21283

30 Rogalska A, Forma E, Ciesielski P, Brys M, Krzeslak A and Marczak A: Effect of metformin on apoptosis induction in ovarian cancer cells. Prz Menopauzalny 13(3): 155-161, 2014. PMID: 26327848. DOI: 10.5114/pm.2014.43817

31 Schuler KM, Rambally BS, DiFurio MJ, Sampey BP, Gehrig PA, Makowski L and Bae-Jump VL: Antiproliferative and metabolic effects of metformin in a preoperative window clinical trial for endometrial cancer. Cancer Med 4(2): 161-173, 2015. PMID: 25417601. DOI: 10.1002/cam4.353

32 Van Cruchten S and Van Den Broeck W: Morphological and biochemical aspects of apoptosis, oncosis and necrosis. Anat Histol Embryol 31(4): 214-223, 2002. PMID: 12196263. DOI: 10.1046/j.1439-0264.2002.00398.xl

33 Elmore S: Apoptosis: A review of programmed cell death. Toxicol Pathol 35(4): 495-516, 2007. PMID: 17562483. DOI: $10.1080 / 01926230701320337$

34 Goldar S, Khaniani MS, Derakhshan SM and Baradaran B: Molecular mechanisms of apoptosis and roles in cancer development and treatment. Asian Pac J Cancer Prev 16(6): 2129-2144, 2015. PMID: 25824729. DOI: 10.7314/APJCP.2015. 16.6.2129

35 Hu S, Ouyang Q, Cheng Q, Wang J, Feng F, Qiao L, Gan W, Shi Y, Wu D and Jiang X: Phenformin inhibits cell proliferation and induces cell apoptosis and autophagy in cholangiocarcinoma. Mol Med Rep 17(4): 6028-6032, 2018. PMID: 29436644. DOI: $10.3892 / \mathrm{mmr} .2018 .8573$

36 Liu Z, Ren L, Liu C, Xia T, Zha X and Wang S: Phenformin induces cell cycle change, apoptosis, and mesenchymal-epithelial transition and regulates the AMPK/MTOR/P70S6K and MAPK/ERK pathways in breast cancer cells. PLoS One 10(6): e0131207, 2015. PMID: 26114294. DOI: 10.1371/ journal.pone.0131207

37 Caraci F, Chisari M, Frasca G, Chiechio S, Salomone S, Pinto A, Sortino MA and Bianchi A: Effects of phenformin on the proliferation of human tumor cell lines. Life Sci 74(5): 643-650, 2003. PMID: 14623034. DOI: 10.1016/j.lfs.2003.07.015

38 Porter AG and Janicke RU: Emerging roles of caspase-3 in apoptosis. Cell Death Differ 6(2): 99-104, 1999. PMID: 10200555. DOI: 10.1038/sj.cdd. 4400476

39 Adamiec-Mroczek J, Zajac-Pytrus H and Misiuk-Hojlo M: Caspasedependent apoptosis of retinal ganglion cells during the development of diabetic retinopathy. Adv Clin Exp Med 24(3): 531-535, 2015. PMID: 26467145. DOI: 10.17219/acem/31805

40 Trousil S, Chen S, Mu C, Shaw FM, Yao Z, Ran Y, Shakuntala T, Merghoub T, Manstein D, Rosen N, Cantley LC, Zippin JH and Zheng B: Phenformin enhances the efficacy of ERK inhibition in NF1-mutant melanoma. J Invest Dermatol 137(5): 1135-1143, 2017. PMID: 28143781. DOI: 10.1016/j.jid.2017. 01.013

Received April 29, 2019

Revised June 2, 2019

Accepted June 4, 2019 